566

\section{PLACENTAL PATHOLOGY IS ASSOCIATED WITH NEUROLOGICAL DEVELOPMENT IN PRETERM INFANTS DURING THE FIRST TWO WEEKS AFTER BIRTH}

\author{
A.M. Roescher ${ }^{1}$, A. Timmer ${ }^{2}$, E.A. Verhagen ${ }^{1}$, \\ J.J.H.M. Erwich ${ }^{3}$, A.F. Bos ${ }^{1}$ \\ ${ }^{1}$ Neonatology, Beatrix Children's Hospital, \\ ${ }^{2}$ Pathology, ${ }^{3}$ Obstetrics and Gynecology, University \\ Medical Center Groningen, Groningen, The \\ Netherlands
}

Background: Little is known about the association of placental pathology and neurological morbidity shortly after birth in preterm infants. Neurological condition during this period can be evaluated by the quality of general movements (GMs).

Aim: To determine whether placental pathology was associated with neurological morbidity in preterm infants during the first two weeks after birth.

Methods: Placentas from 27 singleton, preterm infants (gestational age 26-31 weeks, birth weight 615-2250 grams) were assessed for placental lesions: maternal vascular underperfusion, ascending infection, villitis placentae of unknown etiology (VUE), chronic deciduitis, fetal thrombotic vasculopathy (FTV), meconium associated changes, and other unspecified. The presence of elevated nucleated red blood cells (NRBCs) was also assessed. Neurological morbidity was determined by the quality of GMs as normal, abnormal or hypokinetic, on postnatal day $1-5,8$, and 15 . Additionally a motor-optimality-score (MOS) was obtained.

Results: Examination of the placentas revealed: maternal vascular underperfusion $(n=20)$, ascending infection $(n=9)$, VUE $(n=5)$, chronic deciduitis $(n=9)$, FTV $(n=5)$, meconium associated changes $(n=6)$, and elevated NRBCs $(n=6)$. FTV was associated with abnormal GMs on day $3(p=0.043)$. Maternal vascular underperfusion was associated with low MOS on day $8(p=0.064)$, while ascending infection was associated with high MOS on day $8(p=0.004)$. No association existed between other placental lesions and GMs or MOS.

Conclusions: Placental lesions have limited effects on early neurological morbidity in preterm infants. Only on some days after birth, FTV and maternal vascular underperfusion were associated with neurological morbidity, whereas ascending infection was associated with better quality of GMs.

\section{7}

\section{THE INFLUENCE OF GESTATIONAL AGE (GA) ON TOTAL ABSOLUTE BAND POWER (TABP) DURING THE THREE FIRST DAYS OF LIFE}

E. Schumacher ${ }^{1}$, A. Westvik ${ }^{2}$, P.G. Larsson ${ }^{3}$, R. Lindeman ${ }^{1}$, J. Westvik ${ }^{3}$, T. Stiris ${ }^{1}$

${ }^{1}$ Oslo University Hospital, Ullevål, ${ }^{2}$ Oslo University Hospital, ${ }^{3}$ Oslo University Hospital, Rikshospitalet, Oslo, Norway

Background: Continuous long-term multichannel EEG monitoring of the premature infant is possible. This gives information on the brain's electrical activity during the first days of life. $\mathrm{tABP}$ is a measure of the EEG-background activity. We have shown that automated trimming of the exported data by removal of $5 \%$ of the highest tABP-values correlates well with the visually edited recordings. It is known that the background activity depends on postconceptional age; however, what happens during the first days of life and whether there are GA dependent differences, remains to be demonstrated.

Aim: To analyse EEG background activity of the continuous EEG from day 1 to 3 using tABP and compare two different GA groups during this time period.

Methods: 42 healthy infants with $\mathrm{GA}<31$ wk were included, monitored (NicoletOne monitor) continuously for 3 days soon after birth. 8 EEG 8 electrodes were applied. The infants where divided into two groups Progr $1 \mathrm{GA}[24+0]-[27+6]$ wk and Progr 2 GA [28+0] - [30+6] wk). The mean of tABP medians each day calculated showed a normal distribution and were analysed using ANOVA for repeated measurements.

\section{Results:}

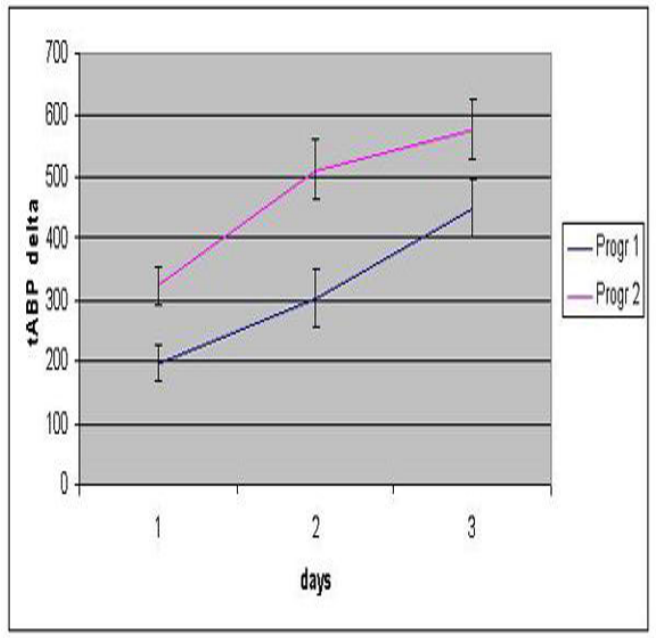

[abstr2ESPR] 
There was a significant difference between the groups throughout the study period. $(\mathrm{P}<0.01)$. However, the increase of tABP from d1-3 within each group did not show any significant difference.

Conclusion: This study shows that the background activity is dependant on GA from birth and that the changes with time after birth are independent GA.

\section{8}

IS THE TORONTO SCORING SYSTEM USEFUL FOR MONITORING THE PROGRESS OF RADIAL NERVE PALSY IN NEWBORN BABIES?
A. Raina1 ${ }^{1}$, M. Sarwar ${ }^{1}$, N. Edi-Osagie ${ }^{1}$, S. Victor ${ }^{2}$
${ }^{1}$ Newborn Intensive Care Unit, Central
Manchester Foundation Trust, ${ }^{2}$ Developmental
and Regenerative Biomedicine Research Group,
University of Manchester, Manchester, UK

Introduction: The Toronto scoring system is used commonly for brachial plexus injuries by physiotherapists and its role in radial nerve palsy is indeterminate.

Aim: To determine if the Toronto scoring system is useful for monitoring the progress and predicting the prognosis of radial nerve palsy.

Methods: The Toronto score assessment for radial nerve palsy was based on the range of motion achieved at the elbow, wrist and finger joints with and without gravity eliminated. Flexion and extension was assessed at each joint and scored from zero (no contraction) to two (full motion). Four term babies born at St. Mary's Hospital, Manchester with isolated radial nerve palsy were reviewed at birth by paediatric physiotherapists. They were given a Toronto score at initial assessment. Subsequently they were reviewed 1-2 weekly and evaluated using the Toronto scoring system. A final evaluation was done prior to discharge.

Results: The Toronto score pre-treatment ranged from 2.3 to 4.9. All four babies received physiotherapy. Physiotherapy was provided with thermoplastic splints for 2 babies and no splints for the remaining two. The Toronto score at the end of 5 weeks of treatment was 10 in three babies. One baby had a Toronto score of 9.3 at 4 weeks of age, when the baby was lost to follow-up.

Conclusion: Toronto score is a useful for monitoring the progress of radial nerve palsy. But in our small series of four patients a low Toronto score at initial assessment did not predict time to recovery.

\section{SINGLE CENTER LONG TERM FOLLOW-UP OF SUPRATENTORIAL ARACHNOID CYSTS IN CHILDREN}

\author{
L. Thewissen ${ }^{1}$, L. De Jong ${ }^{2}$, E. Ortibus ${ }^{3}$, \\ F. Van Calenbergh ${ }^{2}$, G. Naulaers ${ }^{1}$ \\ ${ }^{1}$ Neonatology, ${ }^{2}$ Neurosurgery, ${ }^{3}$ Paediatric \\ Neurology, University Hospitals Leuven, \\ Leuven, Belgium
}

Background and aims: Data on long-term follow-up of supratentorial arachnoid cysts in patients treated conservatively are limited. The perspective of this study was to evaluate the age at diagnosis (prenatally, < $1 \mathrm{y}, 1-18 \mathrm{y}$ ) and long-term educational follow-up of the children diagnosed with a supratentorial arachnoid cyst in our institution. Surgery was restricted to cases with progressive hydrocephalus or intracranial hypertension.

Methods: We retrospectively identified 60 (m 38/f 22) patients between 1984 and 2010 with the diagnosis of a supratentorial arachoid cyst (prenatal $20 \%,<1$ y $27 \%, 1-18$ y $53 \%$ ). We found educational follow-up in 55 cases.

Results: Cysts were classified in 5 groups: sylvian (47\%), midline $(25 \%)$, suprasellar $(10 \%)$, choroid fissure $(8 \%)$, other $(10 \%)$. Associated structural brain anomalies were documented in $13 \%$. Most prevalent were sylvian left cysts $(27 \%)$. Sylvian cysts were diagnosed in $75 \%$ after 1 year. Midline and suprasellar cysts accounted for $83 \%$ of cysts diagnosed prenatally. Mean follow-up was 74 months. $45 \%$ received surgery. $62 \%$ of patients were able to follow normal education, $34 \%$ needed education with special needs $(73 \%$ of the midline cysts). $4 \%$ had multiple disabilities and needed daycare for the mentally disabled.

Conclusions: Location of supratentorial arachnoid cysts differs with time of diagnosis through paediatric life. Late diagnosis $(>1 \mathrm{y})$ is associated with better educational outcome, independent of the need of surgery. Although the prognosis of supratentorial arachnoid cysts is good with a more conservative follow-up, normal education was not reached in $38 \%$. 